\title{
Quantifying pulsed laser induced damage to graphene
}

\author{
Marc Currie, ${ }^{\text {a) }}$ Joshua D. Caldwell, Francisco J. Bezares, Jeremy Robinson, \\ Travis Anderson, Hayden Chun, and Marko Tadjer ${ }^{\text {b) }}$ \\ Optical Sciences Division and Electronics Science and Technology Division, Naval Research Laboratory, \\ Washington DC 20375, USA
}

\begin{abstract}
As an emerging optical material, graphene's ultrafast dynamics are often probed using pulsed lasers yet the region in which optical damage takes place is largely uncharted. Here, femtosecond laser pulses induced localized damage in single-layer graphene on sapphire. Raman spatial mapping, SEM, and AFM microscopy quantified the damage. The resulting size of the damaged area has a linear correlation with the optical fluence. These results demonstrate local modification of $\mathrm{sp}^{2}$-carbon bonding structures with optical pulse fluences as low as $14 \mathrm{~mJ} / \mathrm{cm}^{2}$, an order-of-magnitude lower than measured and theoretical ablation thresholds. [doi:10.1063/1.3663875]
\end{abstract}

Graphene's combination of nearly uniform, broad spectral absorption with $\sim 2.3 \%$ absorption per monolayer is desirable for broadband optical devices. These intrinsic properties make it useful for mode-locking ${ }^{1,2}$ and Q-switching $^{3}$ and also as neutral density filters for $c w$ light and low fluence optical pulses. Practical graphene optical devices require large, uniform, high-quality films measuring at least $5 \times 5 \mathrm{~mm}^{2}$ and deposition on optically compatible substrates. The most promising synthesis methods to meet these criteria are either through $\mathrm{Si}$ sublimation from $\mathrm{SiC}$ (epitaxial graphene $)^{4}$ or chemical vapor deposition (CVD) growth on $\mathrm{Cu}$ substrates, ${ }^{5}$ where it is possible to transfer these graphene films onto different surfaces. ${ }^{6,7}$ In particular, CVD graphene from $\mathrm{Cu}$ is attractive because large-area films are inexpensive, simple to fabricate, and transferable for applications in optics and photonics. In addition, graphene's strong optical absorption enables processing and patterning with lasers. ${ }^{8}$ Current optical patterning approaches depend upon the linear absorption of highly focused $\mathrm{cw}$ laser light causing localized heating, exceeding $1000 \mathrm{~K}$. These results are similar to other localized thermal methods for altering graphene compounds, for example, heating with scanning probes. ${ }^{9}$

In general, laser-induced damage of materials arises from either thermal or non-thermal effects. The $c w$ laser produces thermal damage by absorption of photons and subsequent energy dissipation through phonons, which at sufficient incident optical energy can be violent enough to break bonds. Alternatively, the energy from femtosecond laser pulses is transferred at rates significantly faster than the phonon relaxation time. Thus, hot electrons are created and then cool by giving their energy to phonons on a time scale shorter than thermal diffusion. This ultrafast absorption creates unique energy transfer mechanisms within the solid that depend on the amount of energy absorbed. As such, the absorption of femtosecond optical pulses can produce both thermal and non-thermal effects. Low pulse fluence creates localized heating and causes melting, vaporization, and/or sublimation, while higher fluence produces a large, non-

\footnotetext{
${ }^{a}$ Electronic mail: marc.currie@nrl.navy.mil.

b) Previously with GSG, NA, Crofton, MD 21114 resident at Naval Research Laboratory, Washington DC, 20375.
}

equilibrium electron temperature and through picosecond electron-phonon coupling times provides rapid energy transfer capable of ejecting material. ${ }^{10}$ Continued increases in fluence can eventually induce damage by rapidly ionizing the material and producing a Coulombic explosion. ${ }^{11,12}$ Therefore, during the investigation of photon-induced damage of thin films via pulsed laser sources, both thermal and nonthermal effects must be explored.

In this letter, a quantitative study with a femtosecond pulsed laser establishes the onset of damage in monolayer CVD graphene. Previous efforts broadly estimated the damage thresholds for $c w$ illumination of various graphene compounds during patterning, whereas in this study, $c w$ thermal effects are minimized since the average irradiance levels are orders-of-magnitude below those of the aforementioned $\mathrm{cw}$ patterning work. The laser pulse parameters for the onset of damage are important for examining the performance and limitation of graphene based optic and photonic devices.

Low-pressure CVD growth and transfer of graphene on $\mathrm{Cu}$ foils was carried out using steps described by $\mathrm{Li}$ et $a l^{5}$ Briefly, $\mathrm{Cu}$ foils were heated to $1030^{\circ} \mathrm{C}$ and $\mathrm{H}_{2} / \mathrm{CH}_{4}(\sim 1: 15$, $\left.\mathrm{P}_{\text {total }}<200 \mathrm{mtorr}\right)$ were introduced for $20-30 \mathrm{~min}$. Graphene ( $\sim 1$ in.) was subsequently transferred to 2 -in., double-side polished c-face sapphire substrates by first spin-coating a PMMA film on the graphene/Cu substrate, etching the $\mathrm{Cu}$ away, transferring the PMMA/graphene film to sapphire, and cleaning in solvents. A Ti/Au metal grid was deposited on the surface of the graphene to ensure laser exposure and characterization efforts were spatially correlated.

The graphene samples were illuminated with 50 -fs pulses from an amplified Ti:sapphire laser operating at an $800-\mathrm{nm}$ center wavelength. The 40- $\mu \mathrm{m}$ diameter laser spot produced a somewhat smaller (15-25 $\mu \mathrm{m}$ diameter) damaged region in the graphene film. This occurred at an optical pulse fluence of 14-66 mJ/ $/ \mathrm{cm}^{2}$. The damaged region's size can be understood by incorporating the Gaussian spatial profile of the laser.

A figure of merit for characterizing graphene's structural quality is the comparison of the D $\left(\sim 1350 \mathrm{~cm}^{-1}\right), \mathrm{G}$ $\left(\sim 1580 \mathrm{~cm}^{-1}\right)$, and $2 \mathrm{D}\left(\sim 2700 \mathrm{~cm}^{-1}\right)$ lines within the Raman spectra, as the D line becomes an allowed vibrational mode when an imperfect $\mathrm{sp}^{2}$ graphitic structure is present. ${ }^{13}$ For 
these experiments, three spatial regions are of interest: (I) outside of the laser-irradiated area, (II) within the laserirradiated, damaged-graphene region, and (III) at the boundary between regions I and II. Fig. 1 shows representative spectra from these three regions, where the D, G, and 2D lines are present and vary in intensity depending on location. Clear $\mathrm{G}$ and 2D Raman lines are observed in region I (unirradiated/ undamaged), whereas these lines are completely absent in region II (irradiated/damaged). However, in both these regions, there is a broad line of equal intensity centered at approximately $1356 \mathrm{~cm}^{-1}$, where the D line is expected. The presence of this line in region II without the $G$ and $2 D$ lines implies that we cannot unambiguously assign this broad mode strictly to disordered $\mathrm{sp}^{2}$-carbon. In addition, the intensity of this $1356 \mathrm{~cm}^{-1}$ line is significantly larger in region III (boundary of the irradiated/damaged area), as would be anticipated from heavily damaged graphene. These facts indicate that this increase at the periphery is due to breaking of the $\mathrm{sp}^{2}$-graphene lattice symmetry, but in all three cases, a broad background band is also present that is not related directly to the graphene bonding. Therefore, use of the $D: G$ ratio as a figure of merit was not possible in these samples.

To examine the lateral extent of the optical damage to graphene, Figs. 2(a) and 2(b) show spatial maps of the $G$ and 2D line intensities from a graphene film irradiated with 57$\mathrm{mJ} / \mathrm{cm}^{2}$ pulses (180-mW average power, $1400 \mathrm{GW} / \mathrm{cm}^{2}$ peak irradiance). Similar to Fig. 1, both the $\mathrm{G}$ and $2 \mathrm{D}$ peak intensities disappear in the regions where the graphene was irradiated. Also, the boundary of the irradiated region has a notably larger D line compared to the pristine region, Fig. 2(c). Under these irradiation conditions, the degradation mechanism involves either material ablation or formation of disordered carbonaceous species, presumably due to the breaking of the $\mathrm{sp}^{2}$ hybridized carbon-carbon bonds within the graphene film. The size, shape, and location of these regions in the spatial Raman maps correspond to the regions where a distinct contrast is observed between the irradiated and pristine regions from the scanning electron microscopy

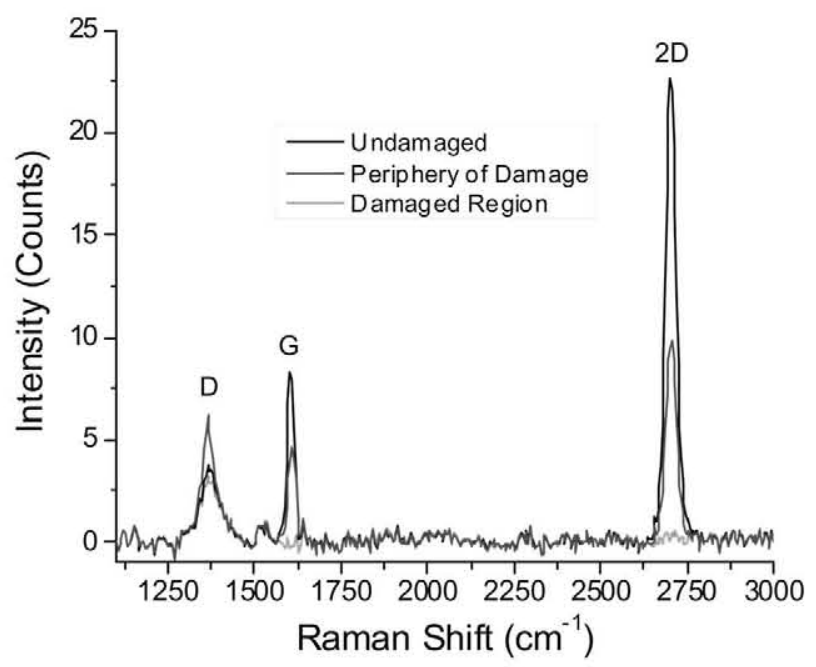

FIG. 1. (Color online) Three micro Raman scans of graphene: (1) unirradiated graphene shows the highest $\mathrm{G}$ and $2 \mathrm{D}$ peaks (black), (2) periphery of the optically damaged graphene shows reduced $G$ and 2D peaks (red), and (3) irradiated/damaged region shows no discernable $\mathrm{G}$ and $2 \mathrm{D}$ peaks (green)

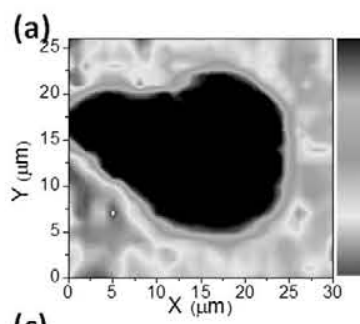

(c)

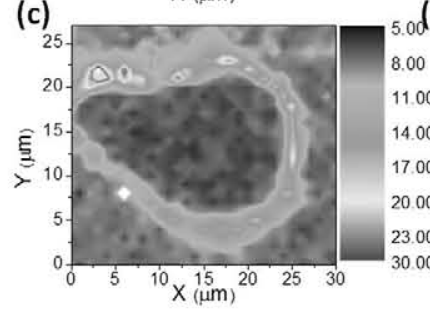

d)
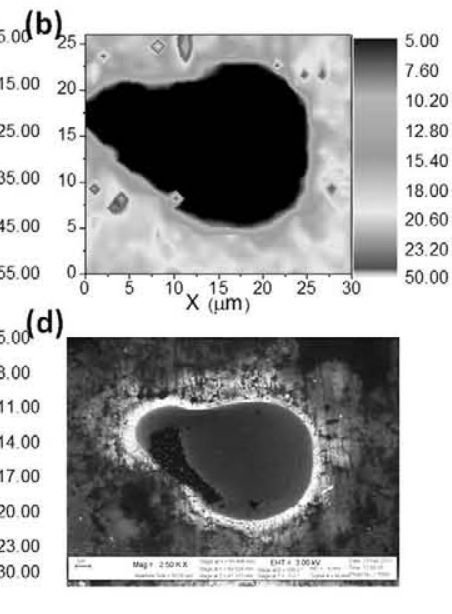

FIG. 2. (Color online) $57-\mathrm{mJ} / \mathrm{cm}^{2}$ optical pulses locally damage a graphene monolayer as show by Raman spatial mapping: (a) 2D line, (b) G-line, and (c) D-line. (d) SEM image of region.

(SEM) imaging, Fig. 2(d). Within the damaged region, there are contrast differences in the SEM image (a darker area in the lower left of the damaged spot); however, the whole irradiated region appears uniform in the Raman maps. As shown in Fig. 2, the presence and size of this damaged region is easily identified, thus both the width and the area may be directly compared to the total incident power and Gaussian profile of the incident laser to access the amount of laserinduced damage.

For all measurements, the Raman $\mathrm{G}$ and $2 \mathrm{D}$ peak intensities decreased in the laser-irradiated regions as compared with the amplitude from the as deposited and un-irradiated graphene measurements. In addition, at fluences greater than $57 \mathrm{~mJ} / \mathrm{cm}^{2}$, we observed a complete loss of the Raman G and $2 \mathrm{D}$ lines within the irradiated region. Variations in the exposure time between 1 and $5 \mathrm{~s}$ had no noticeable impact, implying that the damage induced most likely occurs within the first few pulses. In all experiments, the damaged regions were localized to within the beam diameter of the laser, whereas outside of this region, the graphene remained undamaged, i.e., the Raman signature was consistent with that of the pristine graphene prior to laser irradiation.

The average irradiance used in our experiments had a maximum value of $16 \mathrm{~kW} / \mathrm{cm}^{2}$, much lower than previous reports for $c w$ laser-modification of graphene with $340 \mathrm{~kW} /$ $\mathrm{cm}^{2}$ at $663 \mathrm{~nm}^{8}$ Interactions between $\mathrm{cw}$ lasers and graphene depend upon average irradiance and exposure time. However, graphene's dynamics during and after the optical pulse's absorption include parameters such as optical pulse width, fluence, peak irradiance, repetition rate, and wavelength. Based upon electrical and thermal conductivity, graphitic materials have a $185-\mathrm{mJ} / \mathrm{cm}^{2}$ theoretical damage threshold. ${ }^{14}$ When non-thermal processes are taken into account, there are two theoretically predicted ablation regimes: (1) low-energy, single-layer graphene ablation and (2) higher-energy where nonequilibrium melting and evaporation occurs. ${ }^{15}$ In addition, based upon nonlinear optical measurements, Xing et al. estimate $>300 \mathrm{GW} / \mathrm{cm}^{2}$ for graphene's damage threshold. ${ }^{1}$ In other experiments, Lenner et al. use 100 -fs optical pulses at $800 \mathrm{~nm}$ with $160-\mathrm{mJ} / \mathrm{cm}^{2}$ fluence 

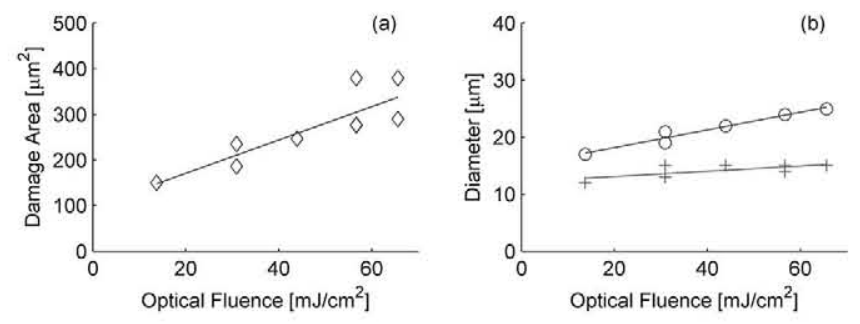

FIG. 3. (Color online) SEM image analysis measured the (a) laser-induced damage area and (b) major $(O)$ and minor $(+)$ axes of an ellipse fit to the damaged area.

to ablate graphite. ${ }^{12}$ Our results illustrate structural damage to graphene at a much lower fluence $\left(14-66 \mathrm{~mJ} / \mathrm{cm}^{2}\right)$ than that used to ablate graphite, shown in the Raman spatial plots, thus, demonstrating our ability to disrupt the $\mathrm{sp}^{2}$ carbon bonding thereby locally modifying graphene.

Analysis of the SEM images, e.g., Fig. 2(d), was used to quantify the optically induced damage area and is shown in Fig. 3(a) as a function of optical fluence. In general, the optical damage manifested itself as a pear-shaped region which was fit with an ellipse. Fig. 3 plots the damage dependence on the major and minor ellipse axes as a function of fluence. The size of the optical damage increased linearly with optical fluence.

In all cases, the pear-shaped damaged area was smaller than the measured 40- $\mu \mathrm{m}$ diameter of our focused laser. The measured diameter ( $1 / \mathrm{e}^{2}$ optical power) of the Gaussian spatial profile was monitored along one axis, thus, the peak irradiance occurs over a diameter smaller than our measured $40-\mu \mathrm{m}$ diameter, and the ellipticity is likely due to an aberration in the focus rather than a physical damage mechanism of the single-layer graphene. The linear fit to the damaged area suggests that our experiments are far above the damage threshold.

The laser pulse parameters for the onset of damage are important for examining the performance and limitation of graphene-based optic and photonic devices, e.g., a 4-GW/ $\mathrm{cm}^{2}$ optical saturable absorber. ${ }^{1}$ We performed a quantitative study with femtosecond optical pulses to establish the onset of damage in CVD grown graphene films transferred onto sapphire substrates. Our average irradiances $\left(3-16 \mathrm{~kW} / \mathrm{cm}^{2}\right)$ were at least an order of magnitude below those used for graphene patterning using $c w$ lasers. We damaged the films with a fluence as low as $14 \mathrm{~mJ} / \mathrm{cm}^{2}$. This fluence is far below those previously found to ablate graphite, and well below theoretical predictions for the damage threshold of graphene, as well as more than two-orders-of-magnitude below the multiphoton ablation threshold $\left(3000 \mathrm{~mJ} / \mathrm{cm}^{2}\right)$ of the sapphire substrate. ${ }^{16}$ Using micro-Raman spectroscopy, we were able to measure the changes in the $\mathrm{G}$ and $2 \mathrm{D}$ lines to determine the extent of the damage and were able to measure the area of the damaged region using SEM imaging. The laser-induced damage was localized without disturbing the surrounding graphene regions demonstrating a potential tool for patterning graphene. Further work is needed to understand the behavior of the carbon bonds during and following laser irradiation and to determine if this process has utility, for example, in local reordering of the bond structure for local bandgap creation in multi-layer graphene.

${ }^{1}$ G. Xing, H. Guo, X. Zhang, T. C. Sum, and C. H. A. Huan, Opt. Express 18,4564 (2010).

${ }^{2}$ H. Zhang, Q. Bao, D. Tang, L. Zhao, and K. Loh, Appl. Phys. Lett. 95, 141103 (2009).

${ }^{3}$ H. Yu, X. Chen, H. Zhang, X. Xu, X. Hu, Z. Wang, J. Wang, S. Zhuang, and M. Jiang, ACS Nano 4, 7582 (2010).

${ }^{4}$ C. Berger, Z. M. Song, T. B. Li, X. B. Li, A. Y. Ogbazghi, R. Feng, Z. T. Dai, A. N. Marchenkov, E. H. Conrad, P. N. First et al., J. Phys. Chem. 108, 19912 (2004).

${ }^{5}$ X. S. Li, W. W. Cai, J. H. An, S. Kim, J. Nah, D. X. Yang, R. Piner, A. Velamakanni, I. Jung, E. Tutuc et al., Science 324, 1312 (2009).

${ }^{6}$ J. D. Caldwell, T. J. Anderson, J. C. Culbertson, G. G. Jernigan, K. D. Hobart, F. J. Kub, M. J. Tadjer, J. L. Tedesco, J. K. Hite, M. A. Mastro et al., ACS Nano 4, 1108 (2010).

${ }^{7}$ S. Unarunotai, Y. Murata, C. E. Chialvo, H.-S. Kim, S. MacLaren, N. Mason, I. Petrov, and J. A. Rogers, Appl. Phys. Lett. 95, 202101 (2009).

${ }^{8}$ Y. Zhou, Q. Bao, B. Varghese, L. A. L. Tang, C. K. Tan, C. Sow, and K. P. Loh, Adv. Mater. 22, 67 (2010).

${ }^{9}$ Z. Wei, D. Wang, S. Kim, S. Kim, Y. Hu, M. K. Yakes, A. R. Laracuente, Z. Dai, S. R. Marder, C. Berger et al., Science 328, 1373 (2010).

${ }^{10}$ X. Liu, D. Du, and G. Mourou, IEEE J. Quantum Electron. 33, 1706 (1997).

${ }^{11}$ M. Lenner, A. Kaplan, and R. E. Palmer, Appl. Phys. Lett. 90, 153119 (2007).

${ }^{12}$ M. Lenner, A. Kaplan, C. Huchon, and R. E. Palmer, Phys. Rev. B 79, 184105 (2009).

${ }^{13}$ F. Tuinstra and J. Koenig, J. Chem. Phys. 53, 1126 (1970).

${ }^{14} \mathrm{~K}$. Sokolowski-Tinten, S. Kudryashov, V. Temnov, J. Bialkowski, D. Von der Linde, A. Cavalleri, H. O. Jeschke, M. E. Garcia, and K. H. Bennemann, Ultrafast Phenomena XII, Springer Series in Chemical Physics Vol. 66, edited by T. Elsaesser, S. Mukamel, M. M. Murnane, and N. F. Scherer (Springer, Berlin, 2001), pp. 425-427.

${ }^{15}$ H. O. Jeschke, M. E. Garcia, and K. H. Bennemann, Phys. Rev. Lett. 87, 87 (2001).

${ }^{16}$ D. Ashkenasi, R. Stoian, and A. Rosenfeld, Appl. Surf. Sci. 154-155, 40 (2000). 